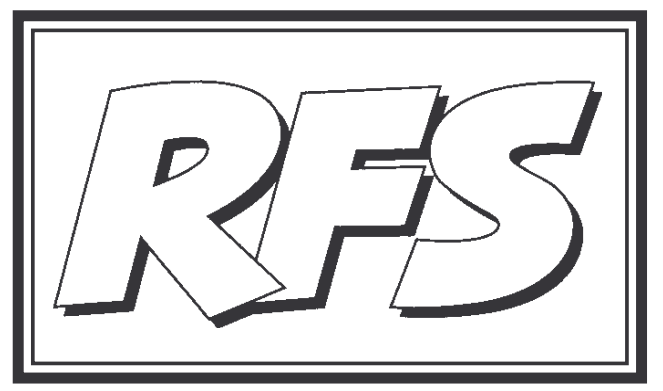

Revista de Fomento Social, 61 (2006), 587-602

\title{
El proyecto de desarrollo rural EXPIDER. Las dificultades en la cuenca del Chanchán (Ecuador) ${ }^{1}$
}

\author{
David J. MOSCOSO SÁNCHEZ2
}

${ }^{1}$ El artículo presenta los resultados de la aplicación del Proyecto EXPIDER a la región citada de Ecuador. Estos resultados fueron compartidos en un seminario organizado por el Instituto de Estudios Sociales Avanzados (IESA-CSIC) tras la culminación de la primera fase del pro yecto. En dicho seminario, celebrado el 27 de enero de 2006 con el título" ¿Nuevos enfoques para el desarrollo rural en América Latina? Reflexiones a partir de la aplicación y evaluación del proyecto EXPIDER en Ecuador, Bolivia y Honduras", además del que subscribe, que se encargó de coordinarlo, participaron José Juan Romero Rodríguez (ETEA) y Encarnación Martínez Navarro (Empresa Pública DAP). Asimismo, esta reflexión también está inspirada en la participación en la Mesa de Expertos sobre Desarrollo Rural y Cooperación, celebrada en la sede de ETEA, en Córdoba, el 19 de abril de 2006.

2 Instituto de Estudios Sociales Avanzados (IESA), Consejo Superior de Investigaciones Cien tíficas (CSIC). Córdoba, España - dmoscoso@iesaa.csic.es 


\section{(Palabras Clave: Proyecto EXPIDER, Desarkollo RuRal, Cuencas hidRográficas, ENFOQUE DE CUENCA.}

KEY Words: PROJECT EXPIDER, RURAL DEVELOPMENT, HydROGRAPHICAL BASINS, BASIN APPROACH)

\section{Introducción}

El agua de las tierras altas está en grave peligro y, con ello, también están en riesgo los valles y los mares. Más de la mitad de la población mundial recibe de las montañas agua, además de energía eléctrica, madera, minerales, tierra cultivable y alimentos. Su gestión y mantenimiento, por tanto, es un reto común, pues nos beneficia a todos: a las poblaciones de estas zonas y a las poblaciones de los valles (Moscoso, 2005 y 2006). Sin embargo, en la actualidad, muchas de las grandes cordilleras del mundo están gravemente amenazadas; más de la mitad de los bosques mueren por la contaminación o la progresiva erosión en los países industrializados y por la deforestación en los países menos favorecidos económicamente. Estos problemas traen asociados otros muchos peligros, tales como la erosión y la consecuente inundación de las tierras bajas, además del incremento de avalanchas y desprendimientos de lodo y desechos.

En las últimas décadas, distintas organizaciones y agencias internacionales han desarrollado estrategias para la gestión del agua en las montañas, a fin de minimizar los riesgos causados por la erosión. Algunos programas han tenido resultados positivos, sobre todo en los países desarrollados (Rodees, 2001; Dammert, 2001; Keek y Hoická, 2004), pero en muchos países en vías de desarrollo resultan casi inviables, pues existen otras dificultades que lo impiden, como la situación de extrema pobreza entre las poblaciones que habitan estas tierras y el mal uso de sus recursos naturales (Ginneken y Thongmee, 1995; Mitchell y Brown, 2002; Raj Kanal y Watanabe, 2006).

Este es el motivo por el que, desde hace años, algunos programas y proyec tos de desarrollo rural puestos en marcha por la FAO, la UNESCO, el Banco Mundial, el Banco Interamericano de Desarrollo, etc., tratan de prevenir el empobrecimiento de las poblaciones y el mal uso de los recursos naturales en las tierras altas y las grandes cuencas hidrográficas de los países en vías de desarrollo. Uno de estos proyectos es EXPIDER que, aunque no se dise ñó con esa finalidad, ni tampoco se ha aplicado con un enfoque de cuenca -pues su objetivo es más global, mejorar la efectividad de los programas 
de desarrollo rural en América Latina, a diferencia de lo que ocurre con otros grandes programas, como MARENA-, lo cierto es que la selección de cuencas hidrográficas para su aplicación implica, directa o indirectamente, una inevitable conexión y, con ello, un trabajo de intervención sobre las problemáticas propias de estas zonas.

El análisis que a este respecto se desarrolla en las presentes páginas se en cuentra estructurado en cinco breves puntos. En el primero se hace referencia, grosso modo, a las condiciones que caracterizan la implantación de políticas de desarrollo rural y cooperación internacional en América Latina, especial mente en nuestros días. En el segundo, se resumen los términos generales que caracterizan al proyecto EXPIDER. En el tercero, se describen las condiciones territoriales, comunitarias y de institucionalidad, de la Cuenca hidrográfica del Chanchán en Ecuador. En el quinto punto se reconstruye la aplicación del-pro yecto EXPIDER en esta zona pilotỏo Por último, en el sexto punto se reflexiona, a modo de conclusión, sobre los límites del proyecto y sobre las oportunidades que éste puede suscitar en cuencas hidrográficas de América Latina.

\section{El contexto de las Políticas de Desarrollo Rural en América Latina}

La mayoría de los especialistas coinciden en que el marco en que tienen lugar las políticas del desarrollo rural en América Latina se caracteriza, en primer lugar, por la enorme heterogeneidad de los países y las regiones de esta área (en términos de contextos políticos, condiciones económicas, estructuras sociales y sistemas culturales), lo que influye de manera determinante en los resultados de cualquier acción de cooperación internacional y desarrollo rural que se emprenda -y, es más, lo que en principio requeriría del diseño de estrategias de desarrollo rural muy específicas a cada territo rio; en segundo lugar, por el hecho de que prácticamente todas las acciones puestas en marcha en materia de desarrollo rural están orientadas a movilizar los recursos técnicos, financieros y humanos en el sector agrícola -sobre todo, en la agricultura de carácter industrial-, una estrategia que en última instancia tiene como principal objetivo el estricto incremento de la produ€

${ }^{3}$ En el equipo de evaluadores participaron una veintena de personas, perteneciendo a la empresa pública Desarrollo Agrícola y Pesquero (DAP) Melchor Guzmán, Encarnación Martínez, Esther Cañizares, Manuel Cuadrado y María José Moreno. En calidad de consultores del Instituto de Estudios Sociales Avanzados (IESA-CSIC) participaron Manuel Pérez Yruela y David Moscoso. Junto a estos, participaron otros colaboradores de diferentes entidades y organizaciones. 
tividad como modelo básico de generación de rentas y, por extensión, del PIB nacional; $y$, en tercer y último lugar, en consonancia con lo anterior, por el escaso protagonismo que en el marco de las políticas y actuaciones de desarrollo rural han tenido las sociedades rurales, quienes supuestamente eran en el pasado -y siguen siéndolo en la actualidad- las principales po blaciones afectadas por los problemas básicos y estructurales a los que, precisamente, tratan de hacer frente dichas políticas y actuaciones.

La mayoría de los enfoques del desarrollo rural predominantes en América Latina hoy, y en el pasado, parecen tener en común estos rasgos. Aquí no ha lugar para profundizar en las distintas etapas y enfoques experimentados en el marco del desarrollo rural en esta región, por lo que remito al lector a revisar los excelentes trabajos al respecto realizados por $\mathrm{M}^{\mathrm{a}}$. E. Cruz Dorén (2002) y O. Plaza (2002), de cuya síntesis es la tabla 1 que se presenta a continuación.

TABLA 1

Enfoques del Desarrollo Rural en América Latina

\begin{tabular}{|l|l|l|}
\hline \multicolumn{1}{|c|}{ Fechas } & \multicolumn{1}{|c|}{ Etapas } & $\begin{array}{l}\text { Enfoques del desarrollo } \\
\text { rural }\end{array}$ \\
\hline $\begin{array}{l}\text { Hasta mitad } \\
\text { del siglo XX }\end{array}$ & $\begin{array}{l}\text { Periodo Hacendal y de Plan } \\
\text { tación }\end{array}$ & Desarrollo Comunal \\
\hline $\begin{array}{l}\text { Años } \\
\text { cincuenta a } \\
\text { setenta }\end{array}$ & $\begin{array}{l}\text { Periodo de Industrialización } \\
\text { Sustitutiva, Reformas Agra- } \\
\text { rias y Colonización }\end{array}$ & $\begin{array}{l}\text { Generación y Transferen- } \\
\text { cia de Tecnología } \\
\text { La Revolución Verde } \\
\text { Reformas Agrarias }\end{array}$ \\
\hline $\begin{array}{l}\text { Años } \\
\text { Setenta a } \\
\text { ochenta }\end{array}$ & $\begin{array}{l}\text { Periodo de Liberalización } \\
\text { Económica: La entrada hacia } \\
\text { la Economía de Mercado }\end{array}$ & $\begin{array}{l}\text { Sistemas de Producción } \\
\text { Desarrollo Rural } \\
\text { Integrado } \\
\text { Congelación del }\end{array}$ \\
\cline { 1 - 1 } $\begin{array}{l}\text { Años noventa } \\
\text { hasta } \\
\text { nuestros días }\end{array}$ & $\begin{array}{l}\text { Periodo de Libre Mercado } \\
\text { con la Intervención del } \\
\text { Estado }\end{array}$ & $\begin{array}{l}\text { Desarrollo Rural } \\
\text { Fondos de Compensación } \\
\text { Social }\end{array}$ \\
\hline
\end{tabular}

Fuente: Elaboración propia. 
La aplicación del proyecto que aquí se analiza, el proyecto EXPIDER, se ubicaría en el marco que caracteriza al último periodo y, en concreto, en el que predomina el enfoque de Desarrollo Rural Integrado (DRf.) Este periodo está caracterizado por la incorporación de algunos países de América Latina a la economía de libre mercado, lo cual ha generado en los últimos años grandes desajustes sociales y dificultades de las empresas latinoamericanas para adaptarse al contexto de libre mercado. Esto, a su vez, se ha traducido en la intensificación de la pobreza y la desaparición de muchas empresas de producción que son absorbidas por otras mayores de capital extranjero. Por ese motivo, el Estado retoma su intervención en el gasto social destinado a programas sociales y de desarrollo rural, desempeñando así un rol más activo en las áreas rurales, sobre todo en su lucha contra la pobreza. Bajo este nuevo enfoque del desarrollo rural se establecerá una doble estrategia para intervenir: una, preferentemente productiva y de prestaciones de servicios orientada a los sectores campesinos que cuentan con una dotación suficiente de recursos productivos $y$, otra, que se enfoca a los sectores de menores recursos y que se orienta a resolver problemas agudos de pobreza rural" (Cruz Dorén, 2002: 111), para lo cual, los programas diseñados se aplicarán en colabo ración con los Ministerios sociales y también se externalizarán a través de terceros (las Organizaciones Internacionales y las ONG). No obstante esta reanudación de los programas de desarrollo rural, las poblaciones rurales han perdido la centralidad que tuvieron en periodos anteriores en las agen das políticas de estos países.

\section{EXPIDER, tras la estela de la Iniciativa Comunitaria LEADER}

EXPIDER (Experiencias de Desarrollo Local Rural en América Latina) consiste en una iniciativa de cooperación técnica regional iniciada a finales del año 2003 por el BID y financiado por el Fondo General de Cooperación de España (FGCE), la Dirección General de Desarrollo Rural del Ministerio de Agricultura, Pesca y Alimentación de España (DGDR-MAPA) y la contraparte local. La primera fase de este proyecto culminó a finales de 2005 y a mediados del 2006 se puso en marcha la segunda fase.

Este proyecto se planteó como objetivo mejorar la efectividad de los pro gramas de desarrollo rural local en América Latina, a través de la asistencia técnica para el fortalecimiento de la institucionalidad local y la adquisición

${ }^{4}$ Para ampliar más información al respecto, véase PlazA (2002: 38). 
de capacidades, utilizando para ello las experiencias de desarrollo rural en España y otros países de la Unión Europea. Concretamente, el interés del proyecto en este tiempo ha sido identificar e incorporar buenas prácticas de desarrollo rural en el marco de la iniciativa comunitaria LEADER en Europa, esperando que pudiera tener un efecto demostrativo para el diseño y la ejecución de programas de desarrollo rural en América Latina.

\section{1. Áreas de aplicación}

Este artículo analiza las condiciones de partida y los resultados de la aplicación del proyecto en el área concreta de la cuenca del Chanchán, en la Cordillera Central de Ecuador. Sin embargo, hubo otras dos zonas piloto sujetas a la aplicación de este proyecto: la mancomunidad de los Valles Cru ceños, en Bolivia, y las sub-cuencas de Reitoca y Verdugo, en Honduras.

Los criterios que se adoptaron para la selección de estas zonas fueron: a) ser zonas elegibles para el Fondo de ayudas a proyectos productivos y nego cios rurales y b) la dimensión de la zona (superficie y población) y su unidad socio-cultural: la vertebración territorial de sus núcleos rurales y urbanos, la dotación de una base de recursos y potencial productivo, la existencia de organizaciones y redes locales con experiencias en acciones de desarrollo y la estabilidad y gobernabilidad de las instituciones locales.

\subsection{Metodología, términos de trabajo y planificación}

En relación con la metodología y el plan de trabajo, la encargada de coordinar la ejecución de las actividades del proyecto EXPIDER en los tres países fue la Unidad de Desarrollo Rural del Departamento de Desarrollo Sostenible del BID. Para dicha ejecución, el BID contó con la colaboración de los gobiernos de las zonas piloto y una amplia red de consultores, agencias y organizaciones, públicas y privadas, locales e internacionales.

Los términos que definieron el trabajo de consultoría local fueron los siguientes: 1) apoyo técnico a agrupaciones locales de desarrollo rural; 2) apoyo técnico a la elaboración de planes locales de desarrollo rural; 3) se guimiento y evaluación de las experiencias piloto de desarrollo local rural; 4) identificación de buenas prácticas de desarrollo rural en el ámbito local.

El marco de aplicación del proyecto contemplaba tres fases diferenciadas: $1^{\underline{a}}$. Análisis de las zonas piloto; $2^{\underline{a}}$. Fortalecimiento de la institucionalidad 
local para el desarrollo rural (OLDER); $3^{\text {a }}$. Elaboración de un Plan de Desa rrollo (PLADER).

\section{La Cuenca Hidrográfica del río Chanchán}

La Cuenca del Chanchán es parte de la cuenca alta del río Guayas (ver mapa 1). Se encuentra en la provincia del Chimborazo, entre la Sierra Central de Ecuador y la costa de Guayaquil. El área comprende cuatro de los nueve

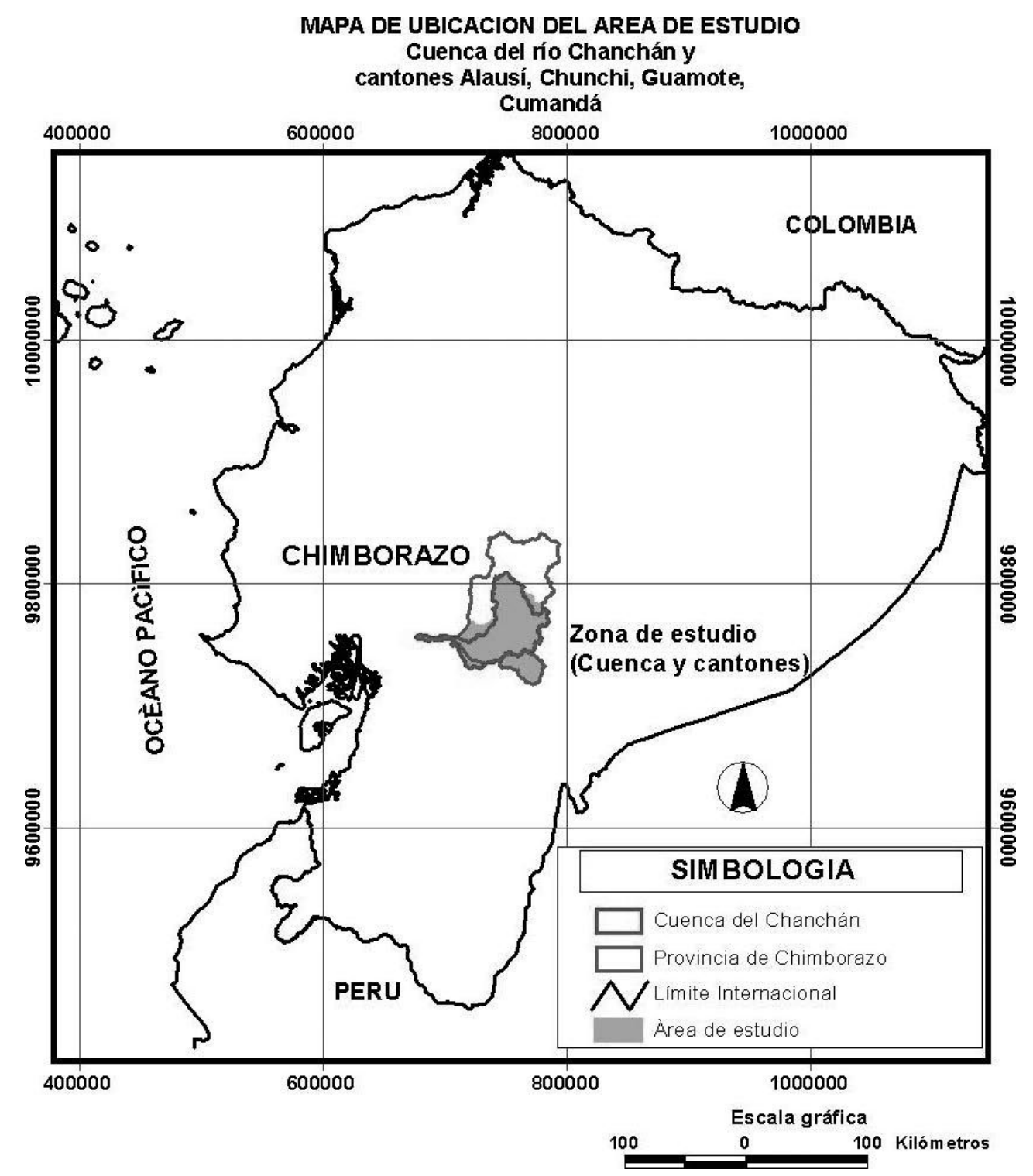

Fuente: Informe final SIG - Chanchán, ECOPAR, 2003. 
cantones de la provincia: Guamote, Alausí, Chunchi y Cumandá. Transcurre entre el nacimiento del río Atapos, en el cantón de Guamote, y la unión de los ríos Chanchán y Chimbo, en el cantón Cumandá. En este recorrido, de una dimensión de aproximadamente $1.500 \mathrm{~km}^{2}$, se presentan diferentes pisos ecológicos, que oscilan entre los 4.500 metros de altitud en el cantón de Guamote y los 400 metros en el cantón de Cumandá. Se trata de un territorio extenso, con una gran variedad climática, ecológica, orográfica y cultural, y que experimenta graves carencias de infraestructuras y equipamientos.

\subsection{Condiciones comunitarias y de institucionalidad}

La población total es de 110.702 habitantes, de las cuales el 80\% están en condiciones de pobreza y el 60\% en extrema pobreza, según el Censo Nacio nal de Población y Vivienda (INEC) de 2001. Destaca su diversidad étnica, caracterizada por grandes conflictos culturales entre mestizos e indígenas (de etnia kichwa y puruhá). También se experimentan graves problemas de desarrollo humano, lo cual se concreta en un elevado grado de analfabetismo, falta de capacitación y problemas de salud. Esto explica la importante tasa de emigración que se da en el territorio. Se trata de comunidades muy desestructuradas, a pesar del amplio número de organizaciones de base que se encuentran activas.

Las condiciones de institucionalidad son débiles: se carece de un sistema administrativo local estable (con continuos cambios políticos y un elevado grado de corrupción e ineficacia administrativa) y predomina una desasisten cia total, por parte de la administración estatal, sobre todo en las pequeñas comunidades de campesinos indígenas. Los responsables políticos locales poseen un escaso grado de capacitación y mantienen graves conflictos políticos entre sí por su condición de amautas (evangélicos) o pachakutis (católicos). Las ONG tienen un gran peso en el desarrollo de estas comunidades y se encuentran muy vinculadas a los partidos políticos y los grupos religiosos, teniendo un gran interés en alcanzar altas cotas de empoderamiento en este área -destacan Fondo Ecuatoriano Populorum Progressio (FEPP), Central Ecuatoriana de Servicios Agrícolas (CESA), Fundación Natura (FN) y Servicio Holandés de Voluntarios (SNV).

La actividad económica se basa esencialmente en la agricultura y la ganadería, que es muy variada (productos tropicales en la parte baja y hof ticultura y pequeñas explotaciones de vacuno y caprino en la parte alta), 
aunque se desarrolla prácticamente en la subsistencia (los habitantes de la zona poseen una media de 2 cabezas de ganado y 2 hectáreas de tierra por familia, que además son poco productivas).

\subsection{El Chanchán desde una visión de cuenca hidrográfica}

Desde los noventa, la cuestión del agua ha ocupado un lugar central en la agenda del desarrollo del país y, en la actualidad, representa uno de los temas prioritarios del debate público. Sin embargo, las principales problemáticas de los recursos hídricos aún no encuentran solución:

- En primer lugar, no tiene solución la demanda de la población pobre (los sectores campesinos) y algunos gobiernos locales, por diseñar políticas públicas que garanticen la equidad ante el aprovechamiento y gobernabilidad de los recursos hídricos. En efecto, en las últimas décadas se ha experimentado una tendencia privatizadora sobre la gestión de este recurso, por parte de empresas transnacionales, que tienen una postura liberalizadora, argumentando que el uso del agua debe privilegiar a la actividad agro-exportadora, porque ésta contribu ye a la generación de divisas. Ante esta tendencia, algunos gobiernos locales y organizaciones campesinas tratan de defender alternativas más democráticas de gestión (con enfoques de participación y equidad, en otras palabras, con enfoque de cuenca), que no cuenta aún con una normativa orientadora estatal. Esta posición alternativa defiende, ante todo, las funciones sociales del agua: la seguridad alimentaria, la auteno mía y la soberanía de las comunidades que habitan estos territorios.

- En segundo lugar, no se soluciona el proceso de erosión que experi menta la parte alta de la cuenca, generado por las malas prácticas agropecuarias, la deforestación y la consiguiente subida de la frontera agrícola, el deterioro de páramos y fuentes de agua, así como el escaso peso y control de la legislación vigente y la ausencia de una propuesta de manejo y gestión sostenible de los recursos naturales. En efecto, la concentración de los sectores pobres (el sector rural y campesino) en las partes alta y media de la cuenca hace que se intensifique de manera incontrolada la explotación de los recursos naturales, de los que dependen económicamente para su subsistencia ante la ausencia de otras alternativas productivas. En conjunto, todo esto puede haber favorecido el origen de algunos desastres naturales (inundaciones y rrimientos de tierra y lodo) producidos en la cuenca del Chanchán. 
En este escenario, la mayoría de los actores sociales y económicos del territorio coinciden en defender que la única solución a estos problemas es la puesta en marcha de instrumentos de participación y concertación social, con capacidad de integrar los intereses y necesidades de los sectores más pobres -que son la mayoría, los sectores campesinos- en las líneas de in tervención pública de manejo del agua. Tal como se indica en el "II Informe de las Naciones Unidas sobre el Desarrollo de los Recursos Hídricos en el Mundo" (UNESCO, 2006), "hay suficiente agua para todos. El problema al que nos enfrentamos en la actualidad es, sobre todo, un problema de gobernabilidad: cómo compartir el agua de manera equitativa y asegurar la sostenibilidad de los ecosistemas naturales. Para ello, parece necesario plantear iniciativas que contribuyan al fortalecimiento de la institucionalidad local y el desarrollo de nuevas actividades económicas, como el turismo natural y patrimonial. «Promover programas integrales de desarrollo hidrográfico a través de la participación efectiva de la poblaci ón local es la clave para evitar que prosiga el deterioro ecológico" (FAO, 2005). Sólo así es posible alcanzar una gestión sostenible de los recursos hídricos.

La visión de gestión de recursos naturales con enfoque de cuenca, en el caso del Chanchán, no se justifica sólo por la interdependencia de las zonas alta, media y baja de la cuenca. Existen otras razones que justifican la puesta en marcha de este enfoque, basado en la participación y concertación social para la gestión de los recursos hídricos:

- En primer lugar, el Chanchán cuenta con importantes lazos históricos y culturales, referidos fundamentalmente a estrategias de intercambio y comercialización. Desde la época precolonial se convirtió en un co rredor en el que tuvieron lugar importantes intercambios económicos con la costa, siendo su eje central Alausí. Esta situación se dio hasta la última década de los años noventa, tras la decadencia del ferrocarril y el posicionamiento del mercado en Guamote, que favoreció los lazos económicos hacia el centro y sur del país.

- En segundo lugar, pese a las claras heterogeneidades en términos de valores culturales como en prácticas económicas que existen entre los cantones, la cuenca del Chanchán es un territorio con una identidad común, en el sentido de que la diversidad cultural es vista aquí como una característica del territorio, considerándose ésta como algo po sitivo. A esa construcción han contribuido históricamente un amplio conjunto de comunidades y actores sociales, con intereses dispares y 
enmarcados en dinámicas sociales particulares. Ello justifica también una acción compartida sobre la gestión de sus recursos naturales, en la que se encuentren representados todos los sectores y actores del territorio, a fin de encontrar solución a la compleja problemática so cioambiental que la caracteriza.

\section{La aplicación del programa EXPIDER}

El proyecto EXPIDER comienza a aplicarse en la zona piloto de la cuenca del Chanchán en marzo de 2004. La contribución del proyecto a la solución de los problemas de la cuenca se concreta en la labor de asistencia técnica para la adquisición de capacidades, desarrollada por los técnicos y consultores, cuyos objetivos eran el fortalecimiento de la institucionalidad local para el desarrollo rural (a través de la creación de las Organizaciones Locales para el Desarrollo Rural, OLDER, homólogas de los Grupos de Acción Local -GAL-eu ropeos) y la elaboración de un plan de desarrollo rural (PLADER).

Antes de la aplicación del proyecto EXPIDER, ya se había iniciado un incipiente proceso de desarrollo en la cuenca del Chanchán, liderado por las principales ONG del territorio, aunque no tenía por finalidad el desarrollo de las comunidades campesinas, sino exclusivamente la gestión de los recur sos hídricos de la cuenca. Los enfrentamientos entre los gobiernos locales y la falta de liderazgo entre los actores que intervenían en el desarrollo de la cuenca dificultó la creación de una Mancomunidad, de modo que cada municipio privilegió sus prioridades.

En este contexto, los trabajos de EXPIDER se fijaron como principal objetivo la creación de una cultura de cooperación y la elaboración de un proyecto de desarrollo compartido entre los municipios que integran la cuenca. Con este fin, los técnicos y consultores del programa trabajaron activamente, mediante labores institucionales, con responsables de los Ministerios de Agricultura, Economía y Bienestar Social de Ecuador, la Prefectura de la Provincia del Chimborazo y las municipalidades de los cantones de la zona piloto. También se trató de establecer relaciones con algunas organizaciones de base (Parlamento Indígena y Campesino, Corporación de Mujeres Rurales de Guamote y Alausí, Corporación de Asociaciones Campesinas Sula, etc.), el sector privado (Cooperativa de Queseros de Guamote, Cooperativa de Crédito y Ahorro de Riobamba, Camping Sillogoto, Cooperativa Ganadera Arte, etc.) y las ONG que trabajaban en la zona -ya señaladas-, a fin de que 
participaran activamente en el proyecto, en especial en la constitución y gestión de la OLDER.

También se desarrollaron sendos talleres de trabajo (entre junio de 2004 y febrero de 2005), donde se definiría y planificaría la filosofía del proyecto EXPIDER, a través de las enseñanzas de la Iniciativa Comunitaria LEADER. Estos talleres perseguían el objetivo de transmitir el funcionamiento de las mancomunidades en España, determinando las directrices de actuación para su constitución en la cuenca y favoreciendo los canales de comunicación entre los diferentes actores. Algunos de estos talleres se desarrollaron en las municipalidades de la Cuenca del Chanchán y un último taller se celebró en Madrid (España), donde fueron invitados los responsables políticos y los actores sociales y económicos de las tres zonas piloto del programa EXPIDER, así como expertos de distintos organismos internacionales (Banco Mundial y BID), técnicos de empresas públicas de desarrollo rural y agrario (Agencia Gallega de Desarrollo Rural -AGADER- y Empresa Pública Desarrollo Agrí cola y Pesquero -D.A.P.-), especialistas del ámbito universitario y científico (Fundación ETEA y Consejo Superior de Investigaciones Científicas -CSIC-) y responsables públicos del Ministerio de Agricultura, Pesca y Alimentación de España. En conjunto, estos talleres contribuyeron a la definitiva creación de la OLDER en la cuenca del Chanchán, denominada formalmente "Corporación para el Desarrollo del Territorio de los ríos Chanchán y Chimbo”. Esta denominación correspondería al hecho de que un cantón vecino a la cuenca del Chanchán, Pallatanga, ubicado en la cuenca del río Chimbo, se unió a los anteriores en el proyecto de mancomunidad en una fase posterior del proyecto.

Otra de las tareas llevadas a cabo fue el análisis del potencial de actividades económicas alternativas, como el turismo, al pensar que esta actividad podría contribuir a la articulación del territorio. Se realizaron varios talleres con el fin de analizar el potencial de la actividad turística en la cuenca del Chanchán, coordinados por especialistas españoles, y se diseñó un plan de turismo para los próximos años. Asimismo, se firmó un convenio de colaboración entre AGADER y la Corporación para el Desarrollo del Territorio de los ríos Chan chán y Chimbo, por el que la agencia española financiaría en los próximos años diversos proyectos de desarrollo en el marco del programa EXPIDER; entre otros, la puesta en valor de los recursos turísticos y el diseño de una campaña publicitaria para atraer a turistas extranjeros a la zona. Con la misma pretensión de favorecer la diversificación de actividades económicas, como es característico de la filosofía LEADER, se invitó a algunos de esos actores 
y responsables políticos a realizar una visita a España para que conocieran algunas acciones demostrativas de desarrollo del turismo rural llevadas a cabo por Grupos de Acción Local (GAL) de la región de Galicia.

\section{Los límites de EXPIDER y el éxito para la gestión de la cuenca del Chanchán}

La pregunta que deberíamos plantearnos, una vez analizado el trabajo realizado por EXPIDER en la zona piloto del Chanchán, es si estas actividades han contribuido de alguna forma a la gestión de los recursos hídricos de la Cuenca. Nuestra opinión es que, pese a no haber actuado directamente sobre las problemáticas ambientales del agua en el territorio, su labor pue de haber tenido una incidencia indirecta aún más positiva al respecto. En efecto, tal como se ha aludido anteriormente, los problemas para la gestión de los recursos naturales en las cuencas hidrográficas de los países en vías de desarrollo han de solventarse sobre la solución de otros problemas de mayor alcance. Entre ellos, mencionamos la creación de instrumentos para la participación y concertación social del conjunto de los actores que hay en el territorio (también, y en especial, de los más pobres, los sectores campesinos) -que ahora tienen una cota de representación, en el caso de la Corporación para el Desarrollo Territorial en los ríos Chanchán y Chimbo-, y los referidos a la diversificación de actividades económicas que contribu yan a poner en valor el patrimonio natural y cultural entre las comunidades que residen en las partes alta y media de las cuencas, a fin de minimizar la sobreexplotación de los recursos naturales.

En definitiva, el paso del proyecto EXPIDER por la Cuenca del Chanchán puede haber contribuido en cierto modo a crear una cultura de cooperación entre los diferentes actores sociales, políticos y económicos del territorio, que además gira sobre una preocupación común: la sostenibilidad de los recursos naturales y, con ello, la supervivencia de las comunidades que residen en el territorio.

EXPIDER, sin embargo, no ha logrado uno de sus objetivos en la zona piloto del Chanchán: la creación del Plan de Desarrollo Rural (PLADER), un requisito muy importante para garantizar compromisos colectivos (en forma de objetivos y estrategias) para el desarrollo del territorio. Este fracaso se explica por las propias dificultades políticas, económicas y sociales, que se dan en la cuenca, sobre las cuales ya hemos hablado. Pero otras dificultades 
tienen mayor relación con la realidad a la que se expone habitualmente la cooperación internacional, a saber:

a) En primer lugar, resulta muy difícil trasladar un proyecto con la metodología propia de la iniciativa europea LEADER a una zona como ésta, pues no se dan las condiciones básicas (una cierta estabilidad política, una masa crítica mínima en la sociedad, la participación de un parte nariado público y privado muy capacitado, etc.) para poner en marcha especificidades tales como la "innovación", el "enfoque ascendente", la "cooperación y el trabajo en red", etc.

b) En segundo lugar, ha de acabarse con los enfoques empleados habi tualmente en la aplicación de programas y planes de cooperación en los países pobres, que se encuentran a menudo excesivamente monopolizados por la labor de los técnicos internacionales, lo cual puede provocar una división de fuerzas. La experiencia ha demostrado que debemos tender hacia un "desarrollo rural de autogestión comunita ria" (Dahl-Østergaard, Moore, Ramírez, Wanner y Bonde, 2003), lo que significa despolitizar la planificación de los proyectos de desarrollo a un nivel local y delegar una mayor responsabilidad técnica sobre los profesionales instalados permanentemente en el territorio. Sólo de esta forma se podría comprometer a un mayor número de actores (especial mente a la población pobre y campesina y las ONG) en la aplicación de este tipo de proyectos de desarrollo rural.

En todo caso, estas dificultades no nos hacen olvidar los logros alcanzados por el proyecto EXPIDER, como la constitución de la Corporación para el Desarrollo del Territorio de los ríos Chanchán y Chimbo, que permite liderar la dinamización de un proceso de diversificación económica en las partes alta y media de la cuenca. Esto tratará de hacerlo involucrando a la mayor parte de los actores locales en las actuaciones de desarrollo que las administraciones ecuatorianas y los organismos internacionales efectúen en la cuenca. Asimismo, su pretensión es orientar este proceso dirigiéndolo hacia las actividades con mayores posibilidades de sostenibilidad ambiental en el largo plazo, a la vez que permitiendo una mejor inserción de las poblaciones rurales a partir de su intercambio en los mercados formales regionales y nacionales. 


\section{Bibliografía}

CRUz D orén, Ma a. E. (2002), "Políticas agrarias y rurales en América Latina. Etapas, enfoques y restricciones e interrogantes", en E. PÉrez CorREA y J. $\mathrm{M}^{\mathrm{a}}$. Sumpsi (Coords.), Políticas, instrumentos y experiencias de desarrollo rural en América Latina y Europa,Madrid, Ministerio de Agricultura, Pesca y Alimentación, pp. 97-122.

Dahl-Østergaard, T., M oore, D., R amírez, V., W anner, M., y Bonde, A. (2003), Desarrollo rural de autogestión comunitaria ¿Qué hemos aprendido?Washington, D. C., Banco Interamericano de Desarrollo (BID).

DAMMERT, L. (2001), "El revestimiento del paisaje: iniciativas de forestación en Islandia", Revista Unasylva, nº. 207, pp.20-21.

FAO (2005), Cuencas Hidrográficas. Disponible en: http://www.alianzamontanas.org/issues/water.html

Ginneken, P.V., Thongmee, U. (1991), "Primeros pasos para el desarrollo integral de la cuenca hidrográfica de Phu Wiang en Tailandia", Revista Unasylva, no. 164 , pp.

KeEK, J. y HoICKÁ, Z. (2001), "Degradación y recuperación de cuencas hidro gráficas de montaña: los montes Jizera en la República Checa", Revista Unasylva, $\mathrm{n}^{\circ} .207$, pp.43-49.

Marghescu, T. (2001), "Restauración de bosques degradados en Tailandia: el caso de Khao Kho", Revista Unasylva, no ${ }^{\circ}$ 207, pp. 52-55.

Mitchell, W. L., y Brown, P. F. (2002), "Los pueblos de montaña: adaptation y pervivencia cultural en el nuevo siglo", Revista Unasylva, $\mathrm{n}^{\circ} .208$, pp. $47-53$.

Moscoso, D. (2005), "Desarrollo rural en zonas de montaña", en E. Moyano (coord.), Agricultura Familiar en España 2005, pp.148-157, Madrid, UPA.

- (2006), "Obstacles and opportunities for mountain development in Anda lusia (Spain)”, Mountain Research and Development, nº. 26/1, pp.81-83.

PlazA, O. (2002): "Perspectivas y enfoques del desarrollo rural: Visión desde América Latina”, en E. Pérez Correa y J. Ma . Sumpsi (Coords.), Políticas, instrumentos y experiencias de desarrollo rural en América Latina y Europa, Madrid, Ministerio de Agricultura, Pesca y Alimentación, pp. 33-64.

Raj Canal, N. y Watanabe, T. (2006), "Abandonment of agricultural land and 
its consequences. A case study in the Sikles Area, Gandki Basin, Nepal Himalaya", Mountain Research and Development, nº.26/1, pp. 32-40.

RHodes, D. (2001), "Rehabilitación de laderas con fuerte declive deforestadas en la costa este de la Isla Norte, Nueva Zelanda", Revistanasylva, nº .207, pp.21-29.

UNESCO (2006), El agua, una responsabilidad compartida. II Informe de las Naciones Unidas sobre el Desarrollo de los Recursos Hídricos en el Mundo. Disponible en: http://www.unesco.org/water/wwap/index_es.shtml 